\title{
Treatment of Lung Metastases from Ewing Sarcoma with Stereotactic Body Radiotherapy rather than Whole-Lung Irradiation
}

\author{
Douglas A Rahn, William Read, Ajay Sandhu
}

\begin{abstract}
Advances in knowledge: Lung metastases from Ewing sarcoma have traditionally been treated with whole-lung irradiation (WLI) and chemotherapy. WLI may unnecessarily treat uninvolved normal lung tissue and increase the risk of toxicity. Stereotactic body radiotherapy (SBRT) has proven to be a safe and effective treatment for primary lung cancer and lung metastases, but there is no significant published description of SBRT in lung metastases from Ewing sarcoma.
\end{abstract}

Implications for patient care: The patient presented in this case report had long-term survival from lung metastases of Ewing sarcoma treated with chemotherapy and SBRT. SBRT may prove to be safer and more effective than whole lung irradiation as a treatment of lung metastases in Ewing sarcoma.

Summary statement: Whether SBRT of lung metastases of Ewing sarcoma can improve outcomes and decrease toxicity compared to whole-lung irradiation, surgical resection or palliative chemotherapy merits further study.

Keywords: Ewing sarcoma, Lung metastases, Whole-lung irradiation, Stereotactic body radiotherapy (SBRT).

How to cite this article: Rahn DA, Read W, Sandhu A. Treatment of Lung Metastases from Ewing Sarcoma with Stereotactic Body Radiotherapy rather than Whole-Lung Irradiation. J Postgrad Med Edu Res 2012;46(1):34-36.

\section{Source of support: Nil}

Conflict of interest: None declared

\section{INTRODUCTION}

Stereotactic body radiotherapy (SBRT) is a well-established treatment for lung metastases, ${ }^{1,2}$ but there is virtually no data about SBRT specifically for lung metastases of Ewing sarcoma (EWS). In the past, whole-lung irradiation (WLI) has been used for EWS with isolated lung metastases ${ }^{3-5}$ as well as prophylactically, although this approach has not gained complete acceptance. The present report describes the use of SBRT to treat a patient with isolated lung metastases of Ewing sarcoma. The discussion focuses on local therapy options in this setting.

\section{CASE REPORT}

A 53-year-old man initially presented with an $11 \mathrm{~cm}$ mass eroding the right anterior first and second rib, which on biopsy proved to be EWS. Further workup revealed no metastatic disease and he was treated with vincristine, adriamycin and cyclophosphamide alternating with ifosphamide and etoposide. He then received radiotherapy to the chest wall mass to $55.8 \mathrm{~Gy}$ in $1.8 \mathrm{~Gy}$ fractions. Minimal lung parenchyma was included in the radiation fields. He had surgical resection of his residual chest wall mass.

A chest computed tomography (CT) 15 months after his original diagnosis revealed 4 new lung metastases, which were hypermetabolic on PET CT scan. He began chemotherapy, but he had progression of lung metastases on chest CT, with a $4.3 \mathrm{~cm}$ cluster in the right lower lobe and a $3.6 \mathrm{~cm}$ cluster in the left lower lobe. Consideration was given for treatment with WLI, but instead we performed SBRT on the above-mentioned bilateral lower lobe lesions to a dose of $40 \mathrm{~Gy}$ in 5 fractions. On post-SBRT PET-CT the treated lesions had regressed and the hypermetabolic signal normalized. Thereafter, he developed several new lung metastases which were likewise treated with SBRT. Altogether he had 5 courses of SBRT with a total of 12 lung metastases treated over 10 months, as summarized in Table 1. Ultimately, he developed progression of the metastases treated in his initial course of SBRT after 12 months of follow-up. The decision was made for him to receive chemotherapy on a clinical trial.

The methods of modern SBRT have been published previously. ${ }^{6,7}$ The patient additionally received numerous regimens of chemotherapy since his diagnosis, including participation in clinical trials although no lesions showed an objective response to any chemotherapy. He had no significant acute or late toxicity from radiotherapy.

\section{DISCUSSION}

The patient presented here survived over 2.5 years since diagnosis of lung metastases from EWS, which were treated with chemotherapy and SBRT. He ultimately had 12 lung metastases treated with SBRT without acute or late toxicity. Given our concerns for the cumulative radiation dose to his lungs, we elected to progressively decrease the bioequivalent dose on his consecutive radiotherapy courses. The majority of the lesions treated demonstrated favorable radiographic response (Figs 1A and B). Ultimately, there was local failure at the highest (initial) dose of $40 \mathrm{~Gy}$ in 5 fractions, although the followup on this course was the longest (12 months). 


\begin{tabular}{lcccccc}
\multicolumn{2}{l}{ Table 1: Description of stereotactic body radiotherapy (SBRT) courses in a patient treated for lung metastases of Ewing sarcoma } \\
\hline Location & $\begin{array}{c}\text { Size } \\
\text { (CM) }\end{array}$ & $\begin{array}{c}\text { Follow-up } \\
\text { (in months) }\end{array}$ & Total RT dose & No. of fractions & $\begin{array}{c}\text { Outcome } \\
\text { (in months) }\end{array}$ & No. of lesions \\
\hline 1. Right lower lobe & 4.3 & 12 & 40 & 5 & Failure at 12 & 5 \\
2. Left lower lobe & 3.6 & 12 & 40 & 5 & Failure at 12 & 3 \\
3. Left hilum & 4.2 & 7 & 40 & 10 & Control at 7 & 1 \\
4. Left lower lobe & 4.3 & 4 & 30 & 6 & Control at 4 & 2 \\
5. Right lower lobe & 1.6 & 2 & 30 & 6 & Control at 2 & 1 \\
\hline
\end{tabular}

In his first description of the disease in $1921,{ }^{8}$ James Ewing noted that it was a particularly radiosensitive tumor. WLI in Ewing sarcoma with isolated lung metastasis has been studied in the past, ${ }^{3-5}$ with the goal of treating both the gross metastases as well as subclinical micrometastases. In fact, these studies suggest that this approach may improve event free survival, but there is reported risk of toxicity, including pneumonitis, restrictive lung disease, abnormal pulmonary function tests and possibly treatment-related death. Whole lung RT may be unnecessary when micrometastatic disease can be controlled by modern chemotherapy. A parallel situation is seen in osteosarcoma, where systemic chemotherapy effectively treats micrometastases present at diagnosis, with surgery used to curatively resect later oligometastatic disease in the lung.

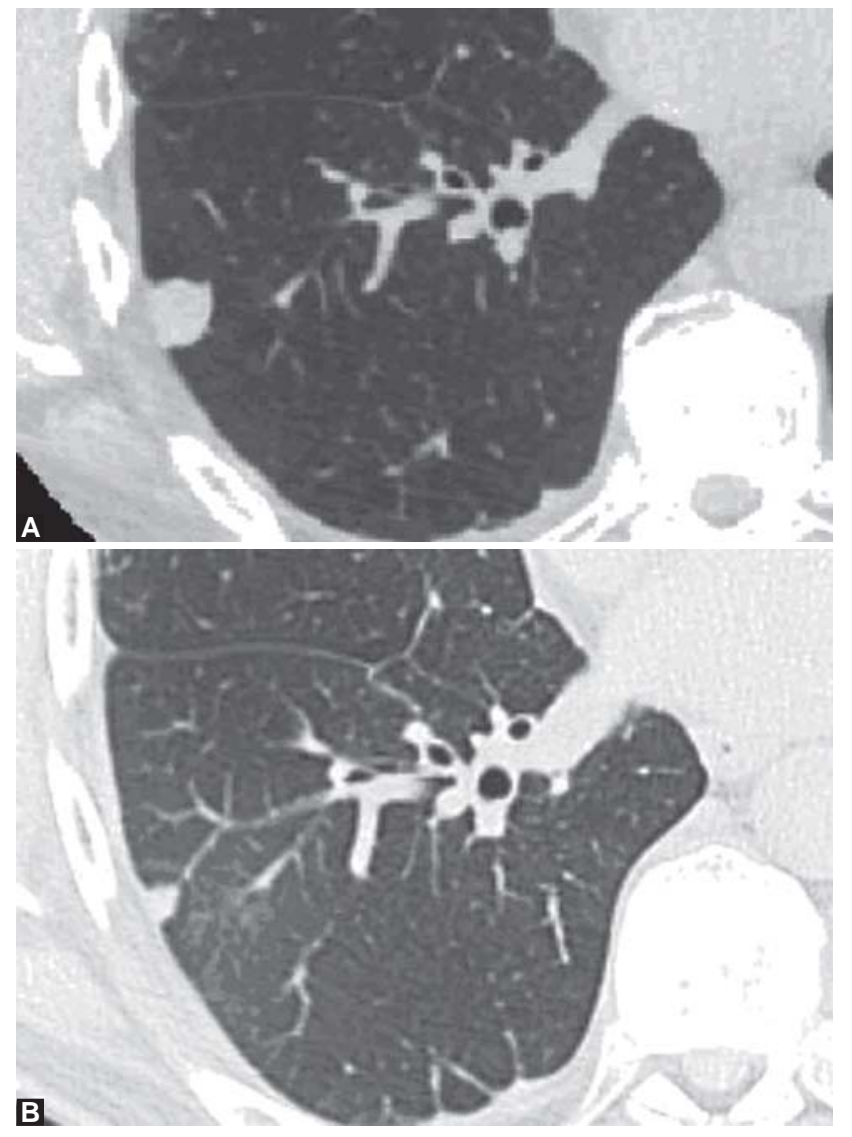

Figs $1 \mathrm{~A}$ and $\mathrm{B}$ : Lung metastasis of Ewing sarcoma treated with stereotactic body radiotherapy seen on axial CT image at pretreatment $(A)$ and 2 months posttreatment, (B) demonstrating regression
Like osteosarcoma, it has recently been reported that surgical resection of pulmonary metastases of Ewing sarcoma can improve survival in selected patients. ${ }^{9}$ Therefore, local treatment of lung metastases of EWS using SBRT may provide comparable benefit to surgery, although there are no published comparisons of these modalities.

Whole-lung irradiation was initially considered for treatment in the present case, but we elected to perform treatment with SBRT to spare as much normal lung tissue as possible. A counter argument would be that WLI may prevent micrometastases from developing into gross disease capable of causing complications. Certainly, the patient's treatment required several courses of SBRT, but this approach was tolerated well without any evident toxicity. Additionally, the doses of subsequent courses of SBRT were of progressively lower bioequivalence, all of which were lower than typical doses for definitive SBRT of primary nonsmall cell lung cancer or SBRT of other histologies of lung metastases. As shown in Table 1, there was eventual failure of some of the treated lesions, but the majority were controlled and showed resolution on PET CT after lower doses of SBRT than the doses used in SBRT for other lung tumors.

SBRT is a well-established treatment of lung metastases from various common tumors, ${ }^{1,2}$ but there is virtually no published data about SBRT specifically for lung metastases of EWS. Patients with progressive lung metastases of EWS on chemotherapy have a poor prognosis, with low-response rates and short-response times. Ewing sarcoma is thought to be a relatively radiosensitive tumor, with radiotherapy demonstrating a high local control rate in the definitive setting. ${ }^{10,11}$ SBRT seems to be an appropriate choice for palliative local therapy of EWS to prevent future airway obstruction, hemoptysis, pain and other complications. Whether SBRT of lung metastases of Ewing sarcoma can improve outcomes and decrease toxicity compared to WLI, surgical resection or palliative chemotherapy merits further study. SBRT could also be used in combination with WLI or as a salvage modality, similar to the paradigm of combining stereotactic radiosurgery and whole-brain radiotherapy for brain metastases. Future clinical research of these comparisons will be valuable as the use of SBRT continues to increase. 


\section{REFERENCES}

1. Okunieff P, Petersen AL, Philip A, et al. Stereotactic body radiation therapy (SBRT) for lung metastases. Acta Oncol 2006;45:808-17.

2. Siva S, MacManus M, Ball D. Stereotactic radiotherapy for pulmonary oligometastases: A systematic review. J Thorac Oncol 2010;5:1091-99.

3. Paulussen M, Ahrens S, Burdach S, Craft A, DockhornDworniczak B, Dunst J, et al. Primary metastatic (stage IV) Ewing tumor: Survival analysis of 171 patients from the EICESS studies. European Intergroup Cooperative Ewing Sarcoma Studies. Ann Oncol 1998;9:275-81.

4. Spunt SL, McCarville MB, Kun LE, Poquette CA, Cain AM, Brandao L, Pappo AS. Selective use of whole-lung irradiation for patients with Ewing sarcoma family tumors and pulmonary metastases at the time of diagnosis. J Pediatr Hematol Oncol 2001;23:93-98.

5. Bölling T, Schuck A, Paulussen M, Dirksen U, Ranft A, Könemann S, et al. Whole-lung irradiation in patients with exclusively pulmonary metastases of Ewing tumors. Toxicity analysis and treatment results of the EICESS-92 trial. Strahlenther Onkol 2008;184:193-97.

6. Nath SK, Sandhu AP, Kim D, Bharne A, Nobiensky PD, Lawson JD, et al. Locoregional and distant failure following image-guided stereotactic body radiation for early-stage primary lung cancer. Radiother Oncol 2011;99:12-17.

7. Nath SK, Sandhu AP, Jensen L, Kim D, Bharne A, Nobiensky PD, et al. Frameless image-guided stereotactic body radiation therapy for lung tumors with 4-dimensional computed tomography or 4-dimensional positron emission tomography/ computed tomography. Clin Lung Cancer 2011;12:180-86.
8. Ewing J. Diffuse endothelioma of bone. Proc New York Path Soc 1921;21:17-24.

9. Letourneau PA, Shackett B, Xiao L, Trent J, Tsao KJ, Lally K, Hayes-Jordan A. Resection of pulmonary metastases in pediatric patients with Ewing sarcoma improves survival. J Pediatr Surg 2011;46:332-35.

10. Evans RG, Nesbit ME, Gehan EA, Garnsey LA, Burgert O Jr, Vietti TJ, et al. Multimodal therapy for the management of localized Ewing's sarcoma of pelvic and sacral bones: A report from the second intergroup study. J Clin Oncol 1991;9:1173-80.

11. Dunst J, Jürgens H, Sauer R, Pape H, Paulussen M, Winkelmann W, Rübe C. Radiation therapy in Ewing's sarcoma: An update of the CESS 86 trial. Int J Radiat Oncol Biol Phys 1995;32: 919-30.

\section{ABOUT THE AUTHORS}

\section{Douglas A Rahn}

Resident, Department of Radiation Oncology, University of California San Diego, USA

\section{William Read}

Associate Professor, Department of Medical Oncology, Imory University, Georgia, USA

\section{Ajay Sandhu (Corresponding Author)}

Clinical Professor, Department of Radiation Oncology, University of California, San Diego, 3960 Health Sciences, Dr MC 0865 La Jolla CA, 92093-0865, USA, e-mail: apsandhu@ucsd.edu 